\section{Earth occultation or coded mask?}

SIR - Zhang et al. ${ }^{1}$ argue that the Earth occultation imaging technique, as used by the BATSE experiment aboard the Compton Gamma-Ray Observatory, holds great promise for long-term, all-sky monitoring of hard X-ray sources, and appears particularly advantageous for space-born applications, since it does not require the use of a "massive" occulting mask which can limit the field of view of high-energy imaging experiments. To demonstrate the capabilities of such a method, Zhang et al. produce images of selected wide fields of the sky, including four images of the region containing the Galactic Centre (GC), taken at different times in the spring of 1992. A comparison between the last two BATSE GC images and nearly contemporaneous images obtained by the French coded aperture telescope SIGMA $^{2}$ aboard the Russian spacecraft GRANAT raises some doubt about the accuracy of the Earth occultation imaging technique where $\mathrm{X}$-ray sources are tightly clustered.

Since March 1990, SIGMA $^{3}$ has provided wide-field sky images in the hard X-ray and soft $\gamma$-ray domain, with an angular resolution of about 15 arcmin. Its coded mask, whose mass (including the support structure) is less than $10 \%$ of the total telescope mass, allows a $16.9^{\circ} \times 18.0^{\circ}$ field of view, contrary to the information reported in Table 1 of ref. 1.

The hard X-ray image of the $\mathrm{GC}$ region presented in part $a$ of the figure combines the data recorded in the $50-100-\mathrm{keV}$ band during two observations made on 17-18 and 18-19, February 1992, the same energy range and in coincidence with the 16-20 February BATSE image. Our map reveals two hard emission excesses above the instrument-detection limit, clearly identified with $1 \mathrm{E}$ 1740.7-2942 and GX 1+4. This latter source, which underwent a dramatic outburst on 17-18 February 1992 (ref. 4) and then dominated the $\mathrm{GC}$ region over the two days covered by the SIGMA observation in the 50-100-kev band, is absent in the related BATSE image, where the only apparent excess concerns 1E 1740.7-2942, which was in a relatively low state at that time $e^{2,4}$. The other SIGMA GC image ( $b$ in the figure) groups three observations performed on 1-2, 2-3 and 4-5 March 1992, which are mainly contemporaneous with the 2--6 March BATSE GC image. Although the two strong excesses detected in the coded mask image are unambiguously identified with GX 354-0 and 1E 1740.7-2942, the Earth occultation technique encounters severe difficulties in disentangling the field. Indeed, the BATSE image suggests a possible contribution of KS 1739-304, even when no hard emission from this latter source was detected by SIGMA ${ }^{2,5}$ (No previous marginal SIGMA detection of KS 1739-304 was claimed by Churazov et $\mathrm{al}^{6}{ }^{6}$, contrary to the information reported in ref. 1.)
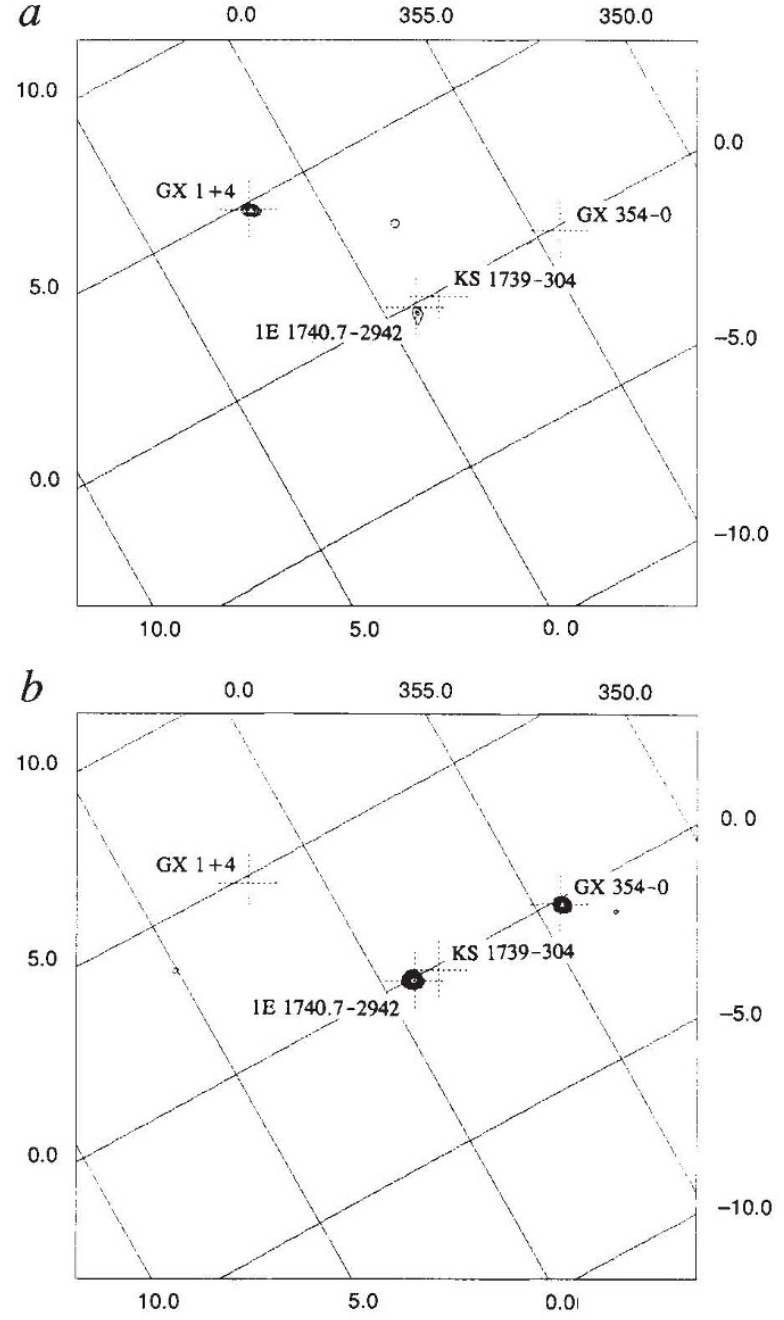

Contour map in galactic coordinates of the GC field, derived in the 50-100-keV band from the data colleted during the 17-19 February (a) and 1-5 March (b) SIGMA GC observations. The contour levels are in units of standard deviations $\langle\sigma\rangle$ over the background mean, starting from $3.5 \sigma$ and further logarithmically disposed at 4.2, 5.0.5.9, 7.1.8.4 and 10.0\%. In $a$, the GX $1+4$ versus $1 \mathrm{E} 1740.7-2942$ SIGMA count ratio is $2.3 \pm 0.7$, while the GX "354-0" versus $1 \mathrm{E} 1740.7-2942$ SIGMA count ratio is $1.01 \pm 0.16$ in $b$.
In consequence, although the Earth occultation imaging technique might appear attractive for all-sky routine survey of the hard X-ray sky, it should be used with great caution for long-term monitoring of hard sources, especially in crowded fields. Moreover, even in the case of the all-sky monitoring task, the limitations of such a technique in terms of source location accuracy may prevent further identification of rapidly varying, hard transient sources. This problem is illustrated by the case of GRS 1716-249, the X-ray nova in Ophiuchus, whose discovery was simultaneously reported by SIGMA $^{7}$ and BATSE $^{8}$, but whose radio, optical and X-ray counterparts were all discovered $^{9-11}$ only because of the small error circle provided at hard X-ray energies by the coded-mask experiment.

\section{Bertrand Cordier, Jacques Paul}

DAPHNIA Service d'Astrophysique,

Centre d'Etudes de Saclay,

91191 Gif-sur-Yvette Cedex, France

\section{Pierre Mandrou,}

\section{Jean-Plerre Roques}

Centre d'Etude Spatiale des

Rayonnements,

9, Avenue du Colonel Roche,

BP 4346, 31029 Toulouse Cedex, France

ZHANG ET AL. REPLY - We are aware of the outstanding scientific observations in high-energy astronomy that are being accomplished by the SIGMA-GRANAT experiment, and we did not mean to imply that the Earth occultation technique can or should take the place of higherresolution coded-mask imagers. Instead we consider Earth occultation imaging as a new and complementary technique for hard X-ray and $\gamma$-ray astronomy. We maintain that its primary advantages are its very wide field (or all-sky) capabilities and its relatively simple flight hardware, as described in our paper ${ }^{1}$.

As pointed out by Cordier et al., it is evident that the two nearby sources, 1E1740.7-2942 and KS 1739-304, are not resolved by the preliminary Earth occultation images shown in our paper. We regret the erroneous reference for the transient source KS 1739-304 in the Galactic Centre region.

Shuang-Nan Zhang, Gerald J. Fishman, B. Alan Harmon \& William S. Paciesas NASA Marshall Space Flight Center, Alabama 35812, USA

1. Zhang, S. N. etal. Nature 366, 245-247 (1993)

2. Cordier, B. et al. Astr. Astrophys. 272, 277-284 (1993)

3. Paul, J. et al. Adv. Space Res. 11, (8) 289-302 (1991).

4. Cordier, B. et al. Astr. Astrophys. Suppl. Ser. 97. $177-180$ (1993)

5. Claret, A et al Astrophys. J (in the press).

6. Churazov, E. et al. Astr. Astrophys. Suppl. Ser. 97 173-176 (1993)

7. Ballet, J. etal. IAU Circ. No. 5874. (1993)

8. Harmon, B. A., Zhang, S. N., Paciesas, W.S. \& Fishman, G. J. IAU Circ. No. 5874 year?

9. Mirabel, I. F., Rodriguez, L. F. \& Cordier, B. IAU Circ. No. 5876. (1993)

10. Della Valle, M etal. B. IAU Circ. No. 5876. (1993).

11. Borozdin, K. etal, IAU Circ. No. 5878. (1993). 